\title{
Les Hémiptères Auchénorhynques de l'Ile de la Réunion
}

\author{
par Jacques Bonfils *, Serge Quilici ** et Bernard ReYNAud *** \\ * 10, rue des Bouvreuils. Aiguefonde. F-81200 Mazamet \\ ** CIRAD/FLHOR (eX-IRFA) - Réunion, Station de Bassin-Martin, Laboratoire d'Entomologie, \\ B.P. 180, F-97455 Saint-Pierre Cedex \\ *** CIRAD/CA-Réunion, Station de Ligne Paradis, F-97410 Saint-Pierre
}

Résumé. - Une mise au point est proposée sur l'ensemble des connaissances de la faune des Homoptères Auchénorhynques de lîle de la Réunion. Par ailleurs, d'abondantes récoltes réalisées depuis 1984, permettent de compléter la liste des espèces présentes dans l'ìle par l'addition de 18 espèces de Fulgoromorpha et 7 espèces de Cicadomorpha. Les affinités biogéographiques de la faune réunionnaise sont discutées.

Summary. - A review is made of our current knowledge of the fauna of Homoptera Auchenorhyncha from Réunion Island. Frequent collects realised since 1984, allow us to complete the list of the island fauna with 18 species of Fulgoromorpha and 7 species of Cicadomorpha. The biogeographic affinities of the island fauna are discussed.

Mots clés. - Hemiptera, Homoptera, Fulgoromorpha, Cicadomorpha, Ile de la Réunion, Faunistique.

D’origine volcanique, l'Ile de la Réunion est située dans l'Océan Indien par $55^{\circ}$ de longitude Est et $21^{\circ}$ de latitude Sud. D'une superficie de $2510 \mathrm{~km}^{2}$, elle est distante d'environ $800 \mathrm{~km}$ de la côte Est de Madagascar, et forme avec les îles Maurice et Rodrigues l'archipel des Mascareignes.

Son climat tropical est tempéré par l'influence des alizés, déterminant une zone «au vent» et une zone «sous le vent», ainsi que par l'altitude. Son relief très accusé est dominé par deux massifs séparés par une plaine d'altitude : celui du Piton des Neiges (3069 m) et celui du Piton de la Fournaise $(2630 \mathrm{~m})$, dont le volcan est encore actif.

L'agriculture a profondément modifié le paysage végétal de l’île. Ainsi, la culture de la canne à sucre a pratiquement éliminé la forêt hygrophile primitive de basse altitude. Toutefois, comparativement à l'île Maurice, il subiste encore de larges lambeaux de la forêt hygrophile de moyenne altitude, d'une grande richesse floristique.

Jusqu'à ces dix dernières années, nos connaissances de la faune des Homoptères Auchénorhynques de la Réunion portaient surtout sur diverses familles du sous-ordre des Fulgoromorpha.

Les Cixiidae de la Réunion ont été étudiés par Synave $(1959,1965)$ puis par Williams (1975b), qui recense au total dix espèces dans l'île, alors que Maurice en compte 32. Cette faune comporte donc peu d'espèces mais celles-ci se diversifient activement, notamment dans le genre Brixia. Celui-ci comprend quatre espèces dont l'une présente quatre sous-espèces, alors qu'à Maurice, où la spéciation a joué depuis beau- 
coup plus longtemps, on recense 26 espèces de ce genre. Les Cixiidae des Mascareignes, étroitement associés à la végétation primaire, sont tous indigènes et, presque sans exception, endémiques d'une île particulière (Williams \& Williams, 1988).

Les Issidae vivent sur les tiges et les rameaux des plantes ligneuses de la végétation indigène. Une première espèce avait été signalée à la Réunion par STÅL, dès 1866. Les trois espèces de l'île sont du genre Tylana et se trouvent aussi à Maurice (WILLIAMS, 1982). Ce genre appartient à une sous-famille essentiellement asiatique (Indonésie, Pacifique) : les Tonginae. Quant aux Derbidae, également inféodés à la végétation forestière, ils comptent 12 espèces aux Mascareignes, dont 5 à la Réunion (Williams, 1976).

A Maurice, les Ricaniidae adultes tendent à se grouper sur certaines espèces d'arbustes ou d'arbres indigènes. On comptait trois espèces de Ricaniidae à la Réunion signalées depuis très longtemps (StÅl, 1866, Melichar, 1898, 1923). Celles-ci sont aussi présentes à Maurice, qui en compte deux autres (Williams \& FenNAH, 1980).

Trois espèces de Tropiduchidae ont également été signalées à la Réunion au siècle dernier (SignORET, 1860, 1862, STÅL, 1866). Une autre a été mentionnée plus récemment par Williams (1981), qui a montré la présence de 9 espèces aux Mascareignes. Seule espèce de Kinnaridae présente dans l'île, Paramicrixia insularis a été décrit de la Réunion par Synave en 1958.

Parmi les Delphacidae, Liburnia centralis a été décrit de la Réunion dès 1860 par Signoret. Diverses espèces ont par la suite été collectées dans l’île : Perkinsiella saccharicida (Kirkaldy, 1903), vecteur de la maladie de Fiji de la canne à sucre, en 1951 (Williams, 1957), puis en 1954, Numata muiri (Kirkaldy, 1907) (Williams \& MaMET, 1954) ainsi que Peregrinus maidis, vecteur de viroses du maïs (Williams \& Williams, 1988). Matutinus lautipes (STÅL, 1858) a été signalé par FenNAH (1972).

La faune des Delphacidae de Madagascar et des Mascareignes a fait l'objet d'une première revue par FENNAH (1964) qui a montré son origine essentiellement africaine. Toutefois, le genre Nesodryas n'existe, en dehors des Mascareignes, qu'aux Marquises et aux îles Hawaï. Les espèces de cette famille sont souvent inféodées à un certain type d'association végétale, plus qu'à un genre ou une espèce de plante-hôte.

Le seul Flatidae de l'île, Flatopsis nivea (Signoret, 1860) était déjà mentionné dans le catalogue de Metcalf (1957).

Le sous-ordre des Cicadomorpha comprend les superfamilles des Cicadoidea et des Cicadelloidea. Des deux espèces de Cicadoidea de 1'île, Abricta brunnea (F.) aurait été récoltée par Nivois dès 1834 (Boulard, 1979) (erreur d'étiquettage ? non retrouvée depuis lors). L'autre espèce, Fractuosella breoni (BoulARD, 1989), bien que déja récoltée par Bréon en 1833, n’a été retrouvée que très récemment (Berne \& Guillermet leg.).

La faune des Cicadelloidea restait très mal connue. Parmi les Cicadellinae, Acopsis viridicans (Amyot \& Serville, 1843) n'a été récolté dans l'île qu'en 1954 (WiLliams \& Williams, 1988). Chez les Deltocephalinae, Etienne \& Roura (1976) signalent la présence de Cicadulina mbila (Naude, 1924), vecteur du maize streak virus; GHAURI (1978) décrit le Typhlocybinae Cerneura delonixia de Maurice et de la Réunion.

Les connaissances sur la faune réunionnaise ont été notablement accrues à la suite de captures effectuées pendant 18 mois à Ligne-Paradis, près de St-Pierre en 1984-85, à l'aide de pièges lumineux, de piège à eau ou à glu par DelPuECH et al. (1986). Cet important travail a permis d'ajouter à la liste des espèces locales douze 
espèces de Fulgoromorpha et 14 de Cicadomopha. Dans cette mise au point, nous nous sommes proposé de rassembler l'ensemble des données dispersées concernant ce groupe d'insectes, et de faire le bilan des collectes réalisées depuis le travail de Delpuech et al. (1986).

\section{MATÉriel et MÉthodes}

Dans le cadre de cet inventaire, diverses méthodes de capture on été utilisées. La majorité des échantillons a été récoltée à l'aide d'un aspirateur à moteur D-Vac ( $\left.{ }^{1}\right)$, d'une cage à cicadelles ou au piège lumineux.

La cage à cicadelles, utilisée en divers milieux herbacés, est du type "piège à émergence» et a été construite selon le modèle de DABRowski (1983). Elle est constituée d'un cadre rigide délimitant un cube de $150 \mathrm{~cm}$ d'arête. Toutes les faces sont recouvertes d'une toile noire, à l'exception de celle en contact avec le sol et de la face «avant", qui est recouverte d'une fine mousseline blanche. Après la pose de la cage sur une formation herbacée, le récolteur s'introduit sous la toile et peut aisément collecter à l'aide d'un aspirateur à bouche les Homoptères qui se regroupent sur la face éclairée.

De nombreux prélèvements ont été effectués de 1985 à 1987 à la cage ou au D-Vac sur des formations herbacées, dans le cadre d'une étude des Auchénorhynques vecteurs de viroses du maïs (REYNAUd, 1988).

Modes et dates de récoltes dans les localités prospectées :

6 Bassin-Martin : fauchage (IV.1985), glu jaune (20-27.XI.1985), D-Vac (16.VI.1987), aspirateur (13.II.1986).

7 Ligne-Paradis : cage (3.VI.85, 25.II, 27.III.86), D-Vac (5.VI, 23.VI, 2.VII.86) aspirateur (28.V, 6.VIII.86). Bassin-Plat : D-Vac (7.VII.1986, 19.V.1989).

9 Etang-Salé : D-Vac (30.VII.1986), glu jaune (8.XII.1987), aspirateur (17.I.1991).

11 Tévelave: D-Vac (26.V.1987).

12 Piton Saint-Leu: D-Vac (26.V.1987).

15 Colimaçons : cage (25.IV, 30.V.1985).

16 Petite-France: cage (25.IV, 30.V, 24.VII, 8.X, 5.XII.1985, 28.I, 25.VII, 27.V, 24.VI.1986).

20 Bois de Nèfles/Saint-Paul : piège lumineux (3.II.1984); aspirateur (3.III.1984).

23 Le Colorado: D-Vac (VII. 1987).

25 La Bretagne : fauchage (III.1986), D-Vac (19.II.1988).

26 Domenjod : piège lumineux (2.IV.1984, III, IV.1985, II.1986, 16.II.1987), aspirateur (1.VI, II.1984).

28 Sainte-Suzanne : aspirateur (15.XI.1983), glu jaune (26.XI.1985-9.IV.1986), D-Vac (25.VI.1986).

30 Bras-Panon: cage (6.VI, 7.XI.1985, 18.II.1986).

31 Bassin-La-Paix : cage (7.XI.1985, 7.II, 18.II, 30.V.1986), D-Vac (30.V.1986, 3.III, 27.XI.1987).

34 Grand-Etang : cage (6.VI.1985).

39 Plaine des Palmistes : piège lumineux (26.XI.83), cage (7.XI.85, 30.V.86), D-Vac (30.V.86, 3.III, 27.XI.87).

43 Le Tampon: D-Vac (23.VII, 13.VIII.1986), aspirateur (18.XI.1987), glu jaune (I.1988, 29.XII.1989).

Des récoltes plus ponctuelles ou plus réduites ont également été effectuées :

— au piège à glu jaune: à Cambaie, du 28.II au 6.III.1984; en zone Nord, de XI.1985 à III.1986; à Convalescence, le 9.IV.1986; à Sainte-Anne de IV à VI.1986; à Saint-François, le 17.IX.1986 ;

— au filet fauchoir; en forêt de Bébour le 7.VI.1991;

(1) D-Vac company, P.O. ; Box 2506, Ventura, C. A. 32002 (USA). 
- à l'aspirateur: Stella, (10.XII.1982); Grand-Bois (19.X, 23.XI.1982); Saint-Benoit (15.XI.1983); Etang-Salé-Les-Hauts (10.XI.1982, 8.VI.1984); Trois-Bassins (10.IV.1986); Saint-Gilles-Les-Bains (10.VII.1986, 9.VII.1987); Forêt de Bélouve (14.XII.1991); Roche Vert-Bouteille (12.I.1992).

La grande majorité des échantillons ont été récoltés sur des cultures ou en zone cultivée, ce qui complète les données antérieures, pour la plupart issues de récoltes en zone de végétation indigène. La situation géographique des localités de captures et de celles qui sont mentionnées dans la littérature est illustrée sur la carte.

\section{RÉSULTATS ET DISCUSSION}

L'examen de plus de 8600 spécimens récoltés de 1984 à 1992 nous a permis d'ajouter à la connaissance de la faune réunionnaise 25 espèces d'Auchénorhynques (18 Fulgoromorpha et 7 Cicadomorpha). Il convient d'y ajouter 7 autres Cicadomorpha nouvellement signalées de l'île mais dont l'identification n'a pu être précisée au niveau spécifique.

En incluant les espèces déjà signalées auparavant dans la littérature, et sans tenir compte des sous-espèces, on dénombre actuellement à la Réunion 95 espèces (62 Fulgoromorpha et 33 Cicadomorpha). Parmi celles-ci, toutefois, la détermination spécifique précise de 8 Cicadomorpha reste à effectuer.

L'analyse de ces résultats montre que certains grands groupes d'Homoptères Auchénorhynques sont absents de la faune insulaire réunionnaise. Parmi les Fulgoromorpha, les familles suivantes n'ont pas de représentants: Achilidae, Dictyopharidae, Eurybrachydae, Lophopidae; de même, les Membracoidea (sensu Deitz), en notant la citation à Madagascar de la seule espèce de Membracidae, Anchon proximum (Signoret), jamais revue depuis lors ; sont absentes également des sous-familles de Cicadellidae connues de l'Afrique et de Madagascar, Acostemminae, Coelidiinae, Idiocerinae, Ledrinae, Ulopinae.

L'analyse de la répartition géographique mondiale de chacune des espèces présentes dans l'île permet une première approche biogéographique de ce groupe à la Réunion, tout au moins pour les familles les mieux représentées.

\section{Fulgoromorpha :}

En ce qui concerne la famille des Delphacidae, nous reprenons les opinions de FENNAH déjà formulées en 1964 (p. 131-132), pour tenter de les appliquer à la faune de la Réunion. Le seul représentant des Alohini, Nesodryas antiope qui a pour hôte le cocotier, aurait pour origine le continent antarctique, tout à fait indépendamment des autres espèces du genre à distribution restreinte à Hawaii, et aux îles Marquises. Les Delphacini comptent à la Réunion 33 espèces, mais cet inventaire n'est pas exhaustif et nous estimons que quelques taxa signalés à l'île Maurice seront probablement trouvés à la Réunion. Au niveau de la répartition géographique des espèces, on peut distinguer cinq groupes :

1 - Les espèces ou sous-espèces à distribution restreinte aux Mascareignes $(3+2)$

2 - Les espèces connues de Madagascar ou des archipels voisins (Seychelles) (3) 


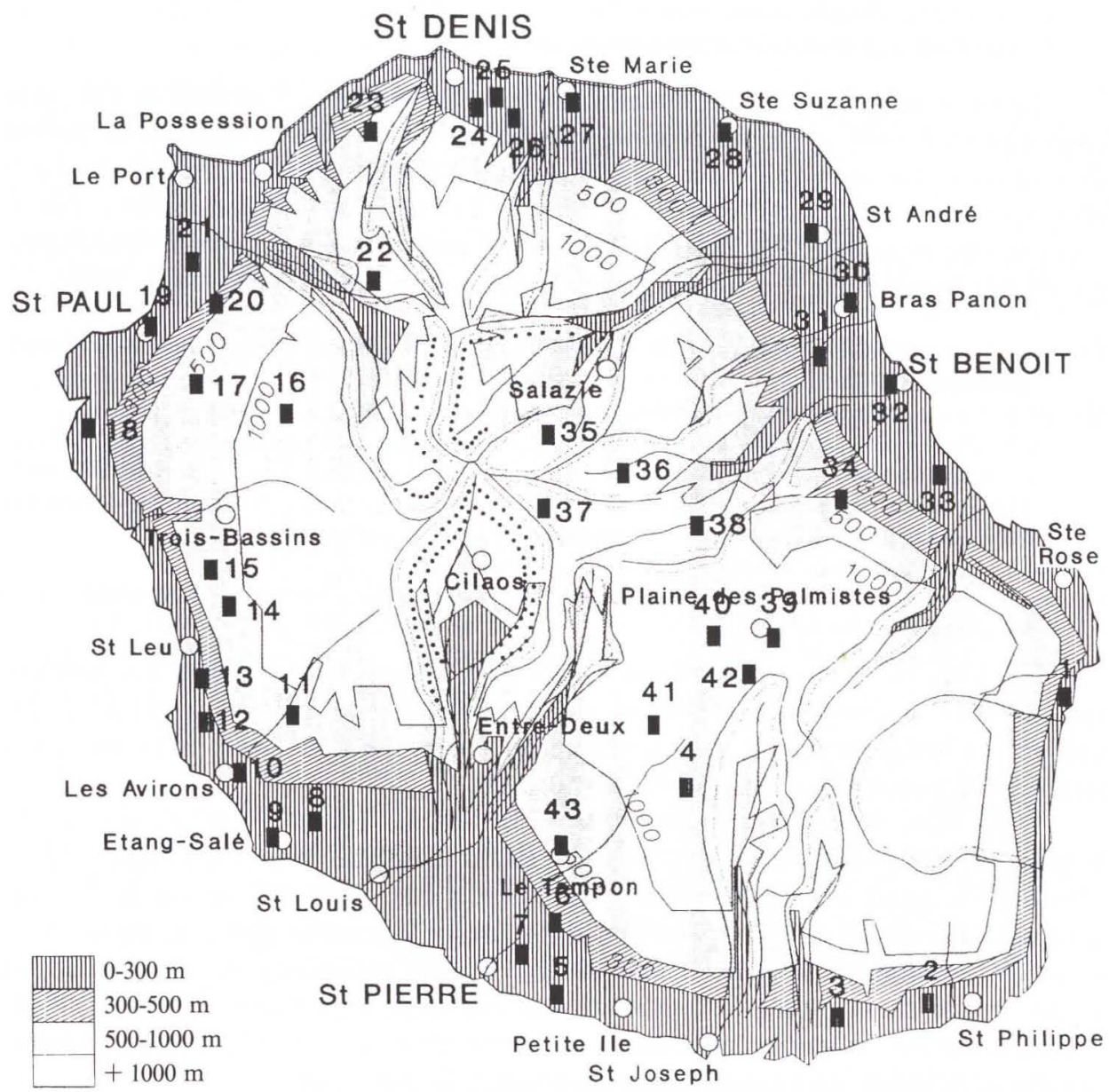

- 1, Bois blane $(80 \mathrm{~m}) ;-\mathbf{2}$, mare Longue $(200 \mathrm{~m}) ;-\mathbf{3}$, Basse Vallée $(250 \mathrm{~m}) ;-\mathbf{4}$, Notre Dame de la Paix $(1600 \mathrm{~m})$; - 5, Grand-Bois $(20 \mathrm{~m})$; - 6, Bassin-Martin $(300 \mathrm{~m})$; — 7, Ligne-Paradis / BassinPlat $(160 \mathrm{~m}) ;-\mathbf{8}$, Etang-Salé-Les-Hauts $(50 \mathrm{~m}) ;-\mathbf{9}$, Etang-Salé $(30 \mathrm{~m}) ;-\mathbf{1 0}$, Les avirons $(200 \mathrm{~m}) ;-$ 11, Le Tévelave $(750 \mathrm{~m}) ;-12$, Piton Saint-Leu $(250 \mathrm{~m}) ;-13$, Stella $((150 \mathrm{~m}) ;-14$, La Chaloupe St Leu $(850 \mathrm{~m}) ;-\mathbf{1 5}$, Colimaçons $(800 \mathrm{~m}) ;-\mathbf{1 6}$, Petite-France $(1400 \mathrm{~m}) ;-\mathbf{1 7}$, Le Guillaume $(600 \mathrm{~m}) ;-$ 18, Saint-Gilles-Les-Bains $(20 \mathrm{~m}) ;-19$, Saint Paul $(50 \mathrm{~m}) ;-\mathbf{2 0}$, Bois de Nèfles/Saint-Paul $(400 \mathrm{~m}) ;-$ 21, Cambaie $(30 \mathrm{~m})$; — 22, Dos d'âne, Roche Vert-Bouteille $(1240 \mathrm{~m}) ;-23$, Le Colorado $(600 \mathrm{~m})$; 24, Le Moufia $(160 \mathrm{~m})$; — 25, La Bretagne $(90 \mathrm{~m}) ;-\mathbf{2 6}$, Domenjod $(150 \mathrm{~m})$; — 27, Sainte Marie $(30 \mathrm{~m}) ;-28$, Sainte-Suzanne $(30 \mathrm{~m}) ;-29$, Saint-André $(100 \mathrm{~m}) ;-30$, Bras-Panon $(50 \mathrm{~m}) ;-31$, BassinLa-Paix (250 m) ; - 32, Saint Benoit (30 m) ; - 33, Sainte-Anne $30 \mathrm{~m})$; - 34, Grand-Etang/Pont Payet (500 m) ; - 35, Hellbourg $(900 \mathrm{~m})$; - 36, Forêt de Bélouve $(1500 \mathrm{~m})$; - 37, Piton des neiges (Coteau maigre, Mare Kerveguen) $(2200 \mathrm{~m})$; - 38, Forêt de Bébour $(1350 \mathrm{~m}) ;$ - 39, Plaine des Palmistes $(1000 \mathrm{~m})$; - 40, Col de Bellevue $(1600 \mathrm{~m})$; — 41, Plaine des Cafres $(1400 \mathrm{~m})$; — 42, Nez de Bœuf $(2130 \mathrm{~m})$; - 43, Le Tampon $(600 \mathrm{~m})$. (Fond de carte : J. P. Jacquemoud-Collet). 
3 - Les espèces distribuées de l’Afrique à l'Océan Indien (18)

4 - Les espèces d'origine asiatique (2)

5 - Les espèces cosmopolites et cosmotropicales (6).

La diversité des formes est remarquable pour la superficie restreinte de l'île, mais le groupement tout arbitraire proposé et sujet à d'éventuelles modifications permet de constater que le «groupe des espèces asiatiques» est le plus pauvre, alors que le plus riche est le "groupe des espèces africaines». Cemus quilicii et Thriambus reynaudi n'ont pu être rapportées à des taxa déjà connus et sont considérées comme endémiques. Ils ont fait l'objet d'une description dans une publication distincte (BonfILS, 1993).

Groupe 1: Cemus quilicii, Oaristes snelli mauritianus, Sogatella colorata nigrior, Tagosodes elpenor, Thriambus reynaudi.

Groupe 2: Cemus leviculus, Nesodryas antiope, Sogatella colorata.

Groupe 3: Afrocoronacella turneri, Eripison snelli, Falcotoya aglauros, Leptodelphax maculigera, Liburnia centralis, Matutinus lautipes, Matutinus pomonus, Numatodes antricauda, Nycheuma dimorpha, Oaristes snelli, Perkinsiella dorsata, Sogatella albofimbriata, S. nigeriensis, Thriambus pondolandensis, Toya bridwelli, Toya demophoon, Toya mastanabal, Toya thomasseti.

Groupe 4 : Cemus pulchellus, Numata muiri.

Groupe 5: Peregrinus maidis, Perkinsiella saccharicida, Sogatella kolophon, Tagosodes cubanus, Toya propinqua, T. tuberculosa.

Les Delphacides se trouvent dominants dans les groupements végétaux herbacés naturels ainsi que dans les agrocénoses. Certains, bien connus pour leurs nuisances aux cultures, se multiplient à la Réunion, principalement dans les zones littorales, apparemment en populations plurispécifiques ouvertes.

D'un point de vue biogéographique, la famille des Issidae ne peut guère actuellement être analysée, son foyer d'origine restant douteux. Toutes les espèces groupées dans le genre Tylana pourraient, selon Williams, 1982: 43, ne pas être congénériques. T. cristata (F.), l'espèce-type du genre, n'appartient pas à la faune de la Réunion. Néanmoins, le genre Tylana, essentiellement indonésien, paraît avoir atteint ici les limites de sa diversification et de son extension vers l'ouest (Fennah, 1964: 132), aucune espèce n'étant signalée du continent africain ni de Madagascar (l'espèce conspersa Schmidt étant considérée comme species inquirenda).

\section{Cicadomorpha :}

Parmi les Cicadellinae, Acopsis viridicans paraît commune au nord, nord-ouest du littoral ainsi qu'en forêt de Bélouve et dans la zone de culture du Géranium rosat : une pullulation fut signalée par le Service de la Protection des Végétaux en 1986 au Guillaume; elle est également commune sur l'île Maurice (Williams \& Williams, 1988 : 4,6). La synonymie entre les genres Acopsis Amyot \& Serville et Ulozena Melichar, établie par Young (1986 : 381) et que nous acceptons, apporte une conclusion satisfaisante aux discussions relatives au statut de ces deux genres. La répartition géographique de l'espèce $A$. viridicans, considérée auparavant comme insulaire, s'étend désormais à Madagascar et au continent africain (Somalie).

Le deuxième genre présent sur l'île de la Réunion, avec Malissiana billosa (Signoret) récolté dans les zones littorales nord, nord-ouest, semble être un apport de Madagascar 
(où le genre compte 5 espèces). Les adultes se capturent sur diverses graminées, comme sur la couverture herbacée des vergers d'agrumes ou sur canne à sucre.

Les Deltocephalinae se trouvent surtout dans les zones littorales et de basse altitude, sur graminées des herbages naturels et des cultures; ils forment des populations parfois abondantes où dominent des espèces à distribution géographique cosmopolite ou pantropicale. Dans les sept genres inventoriés se trouvent plusieurs espèces nuisibles, agents vecteurs de maladies, notamment du maïs (Delpuech et al., 1986). Des quatre espèces du genre Recilia mentionnées, la forme dominante paraît être $R$. mica qui présente une hétérogénéité notable des critères taille ou coloration, selon les stations.

La seule espèce de la sous-famille des Iassinae, Iassomorphus drakensteini a été récoltée jusqu'à présent à la Réunion entre 600 et $1500 \mathrm{~m}$ d'altitude, sur végétation arbustive indigène ou dans les forêts à Acacia heterophylla. Sa distribution géographique couvre une grande partie de la région afrotropicale, l'Afrique du Sud, l'Afrique Centrale, et l'Afrique de l'Est (Linnavuori \& Quartau, 1975 : 26-27).

\section{Conclusions}

Si le présent travail peut contribuer à l'amélioration de nos connaissances sur la faune des Hémiptères Auchénorhynques de la Réunion, il est loin de constituer un inventaire exhaustif de ce groupe pour l'ìle.

En particulier, les prospections futures permettront peut-être de trouver certaines espèces présentes dans d'autres îles de l'Océan Indien. C'est le cas par exemple de Cernea insularis Williams, 1977 (Achilidae), décrite de Maurice. De même, de nouveaux représentants seront certainement trouvés dans des groupes curieusement très peu représentés, comme les Typhlocybinae. Par ailleurs, certains genres dont l'étude se révèle complexe (p. ex. Balchutha, Recilia) nécessiteraient des recherches plus approfondies. Enfin, les deux espèces nouvelles mentionnées dans ce travail font l'objet d'une publication particulière (BONFILS, 1993).

\section{LISTE DES ESPÈCES}

Les espèces endémiques sont indiquées en gras, pour les autres la répartition géographique est mentionnée (pays ou régions, en entier ou abrégés clairement) $($ Mad. = Madagascar, Rod. = Rodriguez, Maur ; = Maurice), pour la Réunion, chaque localité est indiquée par un numéro (voir légende de la carte) suivi s'il y a lieu de la végétation environnante (entre parenthèse) ou du nom français, latin ou créole, de la plante-hôte (souvent abrégé quand ce nom revient souvent) précédée du signe / "sur», puis de la référence bibliographique codée comme suit : B : Boulard, 1989 ; D : Delpuech et al., 1986; DC: Delpech Coll. ; e : Etienne, 1978 ; er : Etienne \& Roura, 1976; f3: Fennah, 1963a ; f4 : Fennah, 1964 ; f2 : Fennah, 1972 ; g : Ghauri, 1978 ; 88 : Melichar, 1898 ; m3 : Melichar, 1923 ; mC: Metcalf, 1957 ; p : Plenet, 1965 ; si0 : Signoret, 1860 ; si2 : Signoret, 1862 ; st : Stal, 1866 ; s8: Synave, 1958 ; s9: Synave, 1959 ; s0 : Synave, 1960 ; s1 : Synave, 1961 ; s5 : Synave, 1965 ; vc: Vuillaume coll.; w7 : Williams, 1957 ; Wa : Williams, 1975a ; Wb : Williams, 1975b ; w6 : Williams, 1976 ; w1 : Williams, 1981 ; w2 : Williams, 1982 ; wF: Williams \& Fennah, 1980 ; wm: Williams \& Mamet, 1954 ; ww : Williams \& Williams, 1988. Les données sans références bibliographiques correspondent aux données nouvelles, acquises au cours de cette étude. 


\section{FULGOROMORPHA}

\section{Fulgoroidea}

Cixindae

Achaemenes quinquespinosus Synave, 1959 : Mad., Maur., Réunion : 2,35,36,38,39,40,41 (s9,wb,w6,ww), 22.

Aselgeoides paludaris Williams, 1975: $39(\mathrm{wb})=A$. insularis Synave, 1959: 35 (s9).

Brixia belouvensis aurata Synave, 1959 : 35,36,38 (s9),4,38,39,40 (wb),36/Fuschia boliviana/Acacia heterophylla.

Brixia belouvensis belouvensis Synave, 1959 : 2,36,38,40,41 (s9), 4,38,40 (wb, B), 16/kikuyu, 22/vég.ind., 36/ Fusc.boliviana, 20 (canne) (lum.).

Brixia belouvensis bipunctata Synave, 1959 : Maur., Réunion : 35,37,38 (s9), 4,38,40 (wb), 22/vég.ind, 36 / Fuschia

Brixia belouvensis tristis Williams, $1975: 42$ (wb).

Brixia costalis Synave, 1959 : 2,35,36,37,38 (s9),4,38,39,40 (wb,ww), 22/vég.ind.

Brixia insularis Synave, 1959: 35,36,38 (s9), 2,4,38,39,40 (wb,ww),20,39 (canne) (lum.), 22/vég.ind, 36/ Fuschia

Brixia lalouettei Williams, 1975 : Mad. Réunion : 35 (wa,ww) = B. stellata Synave, $1959: 2$ (s8,s9,s1).

Eumyndus bistriatus Synave, $1959: 38$ (s9,ww).

Eumyndus pallidus Synave, $1959: 38$ (s9),39 (wb,ww).

Oliarus borbonicus Williams, $1975: 40$ (wb,ww).

Oliarus sanctiphilippi Synave, 1959 : 2,35,36 (s9), 2,40 (wb,ww), 22/vég.ind..

\section{DelPHACIDAE}

Afrocoronacella turneri (Muir, 1926) : C.I., Nigeria, Soudan, Guinée, Afr.sud, Réunion : 7 (canne) (DC), 26. Cemus leviculus Fennah, 1964 : Seychelles, Maur., Réunion : 16/kikuyu,,(DC).

Cemus pulchellus (Distant, 1917) : Sri Lanka, Seychelles, Réunion : 7/maïs (D), 6,25/div.gram.,25 (canne). Cemus quilicii Bonfils, 1993 : 39/Setaria,kikuyu, (D-vac).

Eripison snelli Muir, 1925 : C.I., Nigeria, Cam., Soudan, Rod., Réunion : 7 (canne) (DC), 25.

Falcotoya aglauros (Fennah, 1958) : Sénégal, C.I., Réunion : 7/maïs (D),25,26 (canne),25/canne

Leptodelphax maculigera (Stål, 1859) : C.I., Mad., Maur., Réunion : 7/maïs (D), 15,16/maïs, 6,7/div.gram; 30,31,34/ Setaria sphacelata, 16/kikuyu, 25 (canne).

Liburnia centralis (Signoret, 1860) : Somalie, Réunion : (si0,wb,ww).

Matutinus lautipes (Stål, 1858) : Afr. or., Mad., Réunion : 29 (F2).

Matutinus pomonus (Fennah, 1958) : Guinée, C.I., Tanz., Ouganda, Réunion : 20,25,26 (canne), 31,39/ kikuyu, 1/div.gram (D-vac), 25/canne, 31/ Setaria sphacelata.

Nesodryas antiope Fennah, 1964 : Seychelles (ssp.), Tanz. (WiLson, 1987), Maur., Réunion: /Cocos (E), 26/Cocos.

Numata muiri (Kirkaldy, 1907) = Dicranotropis muiri Kirkaldy, 1907 : Chine, Taïwan, Philippines, Java, Bornéo, Inde, Mad., Maur., Réunion : / canne (B,D), 7/maïs (D), 25,26 (canne), 25/canne (D-vac).

Numatodes antricauda Fennah, 1964 : Soudan, Maur., Réunion : 26 (canne),

Nycheuma dimorpha (Matsumura, 1910) = Dicranotropis capensis Muir, 1926 : Sicile, afrotrop., Maur., Réunion : 7/maïs (D), 20,25,26 (canne), 25/canne

Oaristes snelli mauritianus (Fennah, 1964): Zanzibar (ssp. snelli)(MuIR, 1929), Mad. (ssp. stachi NAST, 1958), Réunion : 7/maïs (D), 30/ Paspalum psittaculum, Seteria sphacelata.

Peregrinus maidis (Ashmead, 1890): pantropical, voire cosmopolite, Seychelles, Mad., Maur., Rod. Réunion : (B, E), / maïs (P), 7/maïs (D), 15,16,34/maïs, 20,25,26 (canne), 6,7/div.gram., 16/Guatemala grass, kikuyu, 30 /Chloris g.,/Setaria sphac., 11/vég.sec; 25/canne (D-vac), 7/manguier (D-vac), 33 (verger d'agrume) (glu).

Perkinsiella dorsata (Melichar, 1905) : Turquie , Chypre, C.I., Nigeria, Ethiopie, Soudan, Tanz., Réunion : 7/mais (D), 7,30/Chloris guayana, 26 (canne) .

Perkinsiella saccharicida (Kirkaldy, 1903) : USA, Perou, Colombie, Equateur, Sud-est asiat., Austr., Hawaii, Afr. s., Comores, Mad., Maur., Réunion: / canne (w7), 25/canne (F4),7/div. graminées, 26 (canne) Cette espèce d'importance économique, vecteur potentiel de la maladie de Fiji, est commune dans l’ìle. Elle fait l'objet d’études détaillées au CIRAD/CA-Réunion. 
Sogatella albofimbriata (Muir, 1926) : Sénégal, C.I., Nigéria , Afr. s., Maur., Réunion : 7/maïs (DC), 30,31,39/ Setaria sphacelata, 30/Paspalum psittaculum, P. dilatatum, 16/Guatemala grass, 34/Hemarthria altissima, 10,25,26 (canne), 31,39/kikuyu, 25/canne

Sogatella colorata nigrior Fennah, 1963 : Seychelles (ssp colorata Distant, 1917), Maur., Réunion : 7/maïs (DC) Sogatella kolophon (Kirkaldy, 1907) : cosmopolite (Asche \& WiLson, 1990), Maur., Rod., Réunion : $7 /$ maïs (DC), 15,16/maïs, 20,25,26 (canne), 25/canne, 30,31,34,39/Setaria sph., 34/Hemarthria alt., $30 /$ Chloris guayana, 30/Paspalum psitt., 16,31/ kikuyu, 6,25/div. graminées, 23/végétation indigène, 11 / vég. secondaire, 19/Calliandra calothyrsus

Sogatella nigeriensis (Muir, 1920) : afrotropical, Iles Glorieuses, Mad., Réunion : 38/div. graminées, 30/ Setaria sphacelata, 16/Guatemala grass, 39/kikuyu, Setaria

Tagosodes cubanus (Crawford, 1914) : Amériques c. et s., Afr.occ. (WILson, 1987), Réunion : 7/maïs (D), 16/maïs, 16,31,39/kikuyu, 39/fétuque, 7/Chloris guayana, 30,31,34,39/ Setaria sphac., 34/ Hemarthria altissima, 39/dactyle, Ray-grass, 25,26 (canne), 7,25/div. gram., 25/canne

Tagosodes elpenor (Fennah, 1964) : Maur., Réunion : 39/kikuyu, Setaria sphacelata, 23/végétation indigène. Thriambus pondolandensis (Muir, 1926) = Dicranotropis stramineus Muir, 1929 : Afr. s; Maur., Réunion : 16/maïs, 20-, 16/kikuyu, 23/végétation indigène, 25 (canne), 25/canne

Thriambus reynaudi, Bonfils, $1993: 26$ (canne)

Toya bridwelli (Muir, 1920) : Nigéria, Sénégal, C.I., Soudan, Israël., Réunion : 7 (DC), 26 (canne), $39 /$ kikuyu, Setaria sphacelata, dactyle, Ray-grass, 16/kikuyu, 30/ Setaria sphac.

Toya demophoon (Fennah, 1963) : Nigéria, Cam., Soudan, Ethiop., Réunion : .16,39/kikuyu, 39/Set. sphac. Toya mastanabal (Fennah, 1969) : Ethiopie (FEnNAH, 1969), Réunion : 7/maïs (D), 16/maiis, kikuyu

Toya propinqua (Fieber, 1866) : cosmopolite, Maur., Rod., Réunion : 7/maïs (D), 6,7,25/div. graminées, 15,16/maïs, 16/kikuyu, 20,25,26 (canne), 25/canne, 30,31/Paspalum sp, 30,39/Setaria sphac., 7/ Chloris guayana, 6/agrumes, 7/manguier, litchi, 28,43 (en verger d'agrumes)

Toya thomasseti (Muir, 1925) : Afr. occ. (Asche, 1988), Soudan, Seychelles, Maur., Rod., Réunion : $22 /$ infloresc. de manguier (vC), 16/maïs (DC), 1/div. gram., 7/maïs, 16,31,39/kikuyu, 30,31,39/Setaria sphac., 30,31/ Paspalum sp., 34/ Hemarthria alt., 39/dactyle, 30/ Panicum max.

Toya tuberculosa (Distant, 1916) : cosmopolite, Réunion : 16/maïs, kikuyu, 39/Setaria sphacelata

Nota: Un certain nombre de spécimens ont été rapportés - avec doute - aux genres ou espèces suivantes : Toya demophoon (Fennah, 1963), Toya mastanabal (Fennah, 1969), Matutinus pomonus (Fennah, 1958). Les espèces suivantes pourraient être trouvées à la Réunion : Nilaparvata maeander (Fennah, 1958), Sogatella gemina (Fennah, 1963), Syndelphax capellanus (Jacobi, 1917), Thymobares longispinus (Muir, 1929).

Derbidae

Aethocauda fusca (Williams, 1976) : Maur., Réunion : 38 (ww)

Aethocauda serrapicis (Williams, 1976) : 40 (w6, ww), 22/ végétation indigène

Diprora lineata (Williams, 1976) : 4,36,40 (w6, ww)

Muirileguatia acuminata (WILlıAMS, 1976) : 36 (w6, ww)

Muirileguatia borbonica (Williams, 1976) : 2 (w6, ww)

FlatidaAe

Flatopsis nivea (Signoret, 1860) : Mad., Maur., Rod., Réunion : /agrumes (MC, P), 26 (canne), 28/agrumes

ISSIDAE

Tylana carinata (F., 1803) : Maur., Réunion : (sT, w2, ww)

Tylana indecora (Stål, 1869) : Maur., Réunion : (w2,ww), 2 (w2), 16,22,23/végétation indigène

Tylana nigromaculata (Williams, 1982): Maur., Réunion : 2 (w2,ww)

KINNARIDAE

Paramicrixia insularis (Synave, 1958) : Maur., Réunion : 2 (s8, ww), 7 (maïs + canne) (DC), 22/vég.indigène

MEENOPLIDAE

Nisia nervosa (Motschulsky, 1863) = Meenoplus atrovenosus (Lethierry, 1888) : cosmopolite (METCALF, 1945), Maur., Réunion: $7 /$ maïs (D), 25,26 (canne) , 1,6,7,25/div. gram., 7,30/Chloris g., 30,31/ Paspalum sp., 30,31,39/ Setaria sphac., 25/canne, 10/vég. sec., 31/ kikuyu 
RiCANIIDAE

Privesa laevifrons (Stål, 1861) : Maur., Réunion : 43 (en verger d'agrumes)

Tarundia cinctipennis (Stål, 1862) : Maur., Réunion : (M3, wF, ww)

Tarundia marginata (Melichar, 1898) : Maur., Réunion : (M3, wF, ww)

Tarundi aservillei (Spinola, 1839) : Maur. Réunion : 2 (M8, M3, st, wF, ww), 22/ végétation indigène

TropiduchidaE

Clardea unicolor (Signoret, 1862) : Maur., Réunion : (si2), 2 (w1), (ww)

Conchyoptera unicolor (Signoret, 1860) : Mad., Maur., Réunion : (si0), 2,4,38,40 (w1), (ww), 22/ vég.indigène

Cuneoceps insularis (Williams, 1983) : Maur., Réunion : 2 (w1, ww)

Idiomyctus notatulus (Stål, 1866) : 2 (st, w1, ww), 22/ végétation indigène

Numicia dorsalis (Jacobi, 1917) : Sierra Leone, Zaïre, Mad., Réunion : 7/maïs (D), 25,26 (canne), 25/canne, $11 /$ vég. s.

\section{CICADOMORPHA}

Cicadoidea

TiBiCENIDAE

Abricta brunnea (F.) : Maur.?, Réunion? (B, ww)

Fractuosella breoni, (BOULARD, 1989) : 3,34 (в)

\section{Cicadelloidea}

Cicadellidae Agallinae

Austroagallia caboverdensis (Lindberg, 1958) : Afrotropical (WeBB, 1980), Maur., Réunion : 7/maïs (D), 1/div. graminées, 25,26 (canne), 30/Paspalum psitt., Setaria sphac., 25/canne, div.gram., 18/melon,

28 (en verger d'agrumes), 16/maïs, 43/Murraya paniculata

Igerna sp. : 6,20/div. graminées, 7,15,16/maïs, 25/canne, 43/Murraya paniculata

\section{Cicadellinae}

Acopsis viridicans Amyot \& Serville, 1843 = Ulozena lineatocollis (Signoret, 1854): Somalie, Mad. Maur., Réunion: (ww), 17/géranium rosat (sPC Coll.), 14/géran. rosat, 36/Acacia het., 11/vég. sec., 36/ div. gram.

Malissiana billosa (Signoret, 1860): Mad., Réunion : 30,31/Setaria sphacelata, 30,31/Paspalum sp., 32/ div. graminées, 25 / canne, 33 en verger d'agrumes, 28 en verger d'agrumes

\section{Deltocephalinae}

Afrosteles distans (Linnavuori, 1959) (det. M.R. Wilson) : Argentine, Afr. s., Maur., Réunion : 31,34,39,43/ div. graminées, 43/fraisier, 31,39/Setaria sphacelata, kikuyu, 34/ Hemarthria, 39/dactyle

Balclutha hebe (Kirkaldy, 1906) : Nigéria (MEdLER, 1980), Réunion : 7/maiis (D), 25,26 (canne), 15,16/ maïs, 6,7/div. gram., 30/Paspalum psitt., 16/kikuyu, 12/Solanum auriculatum, 7/manguier, litchi

Balclutha incisa (Matsumura, 1902) : cosmopolite, Maur., Réunion : 1/div. graminées, 7/maiis, 39/kikuyu

Balclutha punctata (F., 1775) : cosmopolite, Réunion : 20,26 (canne)

Balclutha rosea $($ Scott, 1876) $=$ B. frontalis (Ferrari, 1876) cosmopolite, Maur., Réunion : 7 /maïs (D), 20,25 (canne), 30/ Setaria sphacelata

Balclutha rufofasciata (Merino, 1936) : cosmopolite, Réunion : 7/maïs (D), 25 (canne)

Balclutha saltuella (Kirschbaum, 1868) : cosmopolite, Réunion : 7/maïs (D), 39/kikuyu, 26 (canne), 25/ canne, 11 / végétation secondaire

Balclutha aff. auranticula (Naude, 1926) : Afr. .s., Swaziland, Réunion : 7/maïs (DC)

Cicadulina mbila (Naude, 1924): Inde, Moyen Orient (Rose, 1983), afrotropical, Maur., Réunion : (ER), 7/maïs (D), 15,16/maïs, 25,26 (canne), 25/canne, 16,31/kikuyu, 7,30,31,34/Setaria sphacelata, 7,25,39/div. graminées, 7/Cinchrus, 6/agrumes, 7/litchi, manguier

Cicadulina storeyi (China, 1936) (3) = C. triangula (Ruppell, 1965) : Egypte, Nigeria, Cam., Tanz., Soudan, Zimbabwe., Maur., Réunion: 7/maïs (D), 26 (canne), 25/canne, 31,34,39/Setaria sphac., 34/ Hemarthria alti., 31/ kikuyu, 23/végétation indigène, 33 en verger d'agrumes 
Exitianus capicola (Stål, 1855) : cosmopolite, Maur., Réunion : 7/maïs (dc), 30/Setaria sphacelata, 26 (canne), 25/canne, 16/kikuyu

Exitianus frontalis (Distant, 1917) : Afr.s., Mad., Réunion : 7/maïs (D), 20,25,26 (canne), 25/canne, 23/ végétation indigène, 30,31,34,39/Setaria sphacelata, 15,16/maiis, 6,7,15,25,38,39/div. graminées, 16,31,39/ kikuyu, 34/ Hemarthria altissima

Nesoclutha erythrocephala (Ferrari, 1882) : cosmopolite, Réunion : 7/maïs (D), 7/Chloris guayana (D), 16/ kikuyu, maïs, 23/végétation indigène

Orosius orientalis (Matsumura, 1914) $=$ O. albicinctus (Distant, 1918) : oriental, afrotropical, Réunion : $7 /$ maïs (D), 9 (végétation mixte)

Recilia mica (Kramer, 1962): Afrique occ., Réunion: 7/maïs (D), 15,16/maïs, 26 (canne), 25/canne, div.gram., 16/kikuyu, Guatemala grass, 7/Chloris, div. graminées, 30,31/Paspalum sp., 30,31,34,39 / Setaria sph., 34/ Hemarthria alt., 6,38,39/div. graminées, 39/dactyle, kikuyu, 31/kikuyu

Recilia sp. «B» (? ismenias Linnavuori, 1969) : 7/maïs (DC), 20,26 (canne), 16/kikuyu

Recilia sp. «C» (? shaeuffelei Heller \& Linnavuori, 1968): 7/maïs, herbages ( (DC), 30/ Panicum max./ Chloris g.

Recilia sp. «D»: $7 /$ maïs (DC)

Nota : L'abondance des Recilia récoltés et leur diversité nécessiteraient une étude approfondie.

Hecalinae

Glossocratus afzelii (Stål, 1854) : afrotropical, Mad., Réunion : 7/maïs (D), 31/kikuyu, 23/vég. ind., 26 (canne)

IASSINAE

Iassomorphus dr. drakensteini (Naude, 1926) : afrotrop. soudanais, Réunion : 22,23/vég. ind., 36/Acacia heter.

MACropsinae

Macropsis sp. : 7/maïs (D), 9 en verger de Citrus, 25/canne, 18/manguier

Penthiminae

Penthimia sp. (il s'agit vraisemblablement de l'espèce à laquelle Evans, 1959, fait allusion p. 448 : depuis 30 ans, le statut de cette espèce n'est toujours pas élucidé) : 7 (culture maïs) (D), 5,6,8,13,20,22,26/ manguier, 5,7/avocatier, 26 (canne), 25/div. gram., 7/cocos

Penthimiola bella (Stål, 1855) : afrotropical, Iles du Cap Vert, Mad., Réunion : 7/maïs (D), 6,28/agrumes, 23/vég. ind., 22 en verger de manguier, 25/canne, 9/Murraya paniculata

TyPHLOCYBINAE

Cerneura delonixia (Ghauri, 1978) : Maur., Réunion : 18,24,27/flamboyant (G, ww), 7 (DC), 35/flamboyant, 9,43 / Murray a panic.

Empoasca dolichi (Paoli, 1930) : Afrique s. \& occ., Réunion : 20,25 (canne), 25/canne, div.gram., 7/litchi, $16 /$ mais

Empoasca sp., groupe signata (Haupt, 1927a) : E. signata: Inde, Egypte, Israël, Liban, Zaïre, Réunion : 9/vég. sec.

Xestocephalinae

Xestocephalus sp. : 7 (en zone de culture maïs) (CD), 43 (en verger d'agrumes)

\section{RÉFÉRENCES BIBLIOGRAPHIQUES}

Asche M., 1988. - Delphacidae from Côte d'Ivoire (Homoptera, Fulgoroidea). Revue française d'Entomologie (N.S.), 10 (2) : 15-231.

Asche M. \& M. R. Wilson, 1990. - The delphacid genus Sogatella and related groups: a revision with special reference to rice associated species (Homoptera: Fulgoroidea). Systematic Entomology, 15 : 1-42. 
Bonfils J., 1993 - Deux espèces nouvelles de Delphacides de la Réunion (Hemiptera: Delphacidae). Bulletin de la Société entomologique de France, 98 (3) : 223-226.

Boulard M., 1979. - Révision de la faune cicadéenne des îles Maurice et Rodrigues. Bulletin de la Société entomologique de France, 84 : 22-47.

— 1989. - Une nouvelle espèce de cigale de La Réunion (Tibicenidae, Gymnotympanini) (Hom. Cicadoidea). Bulletin de la Société entomologique de France, 93 (5-6) : 129-132.

Dabrowski Z. T., 1983. - Identifying and collecting Cicadulina for maize streak resistance screening. I.I.T.A. Research Briefs, 4 (4) : 2-3.

Delpuech I., Bonfils J. \& Leclant F. 1986. - Contribution à l'étude des virus du maïs transmis par les Homoptères Auchénorhynques à l'ile de la Réunion. Agronomie, 6 (6) : 549-554.

ETIENNE J., 1978. - Arthropodes nuisibles aux cocotiers à la Réunion. In : «Rapport annuel IRAT-Réunion 1978», pp. 29-31.

ETIEnNe J. \& Roura A., 1976. - Insectes nuisibles nouveaux pour la Réunion. In : «Rapport Annuel IRAT-Réunion 1976»; 166 pp., p. 43.

Evans J. W., 1953. - Les Cicadellidae de Madagascar (Homoptères). Mémoires de l'Institut Scientifique de Madagascar, Série E, 4 : 87-137.

1959. - Quelques nouveaux Cicadellides (Homoptères) de Madagascar. Observations sur la signification évolutive de la spéciation dans le genre Coloborrhis Germar. Mémoires de l'institut scientifique de Madagascar, série E, XI : 481-507.

Fennah R. G., 1954. - The higher classification of the family Issidae (Homoptera : Fulgoroidea) with descriptions of new species. Transactions of the Royal entomological Society of London, 105 : 455-474.

— 1958. - Fulgoroidea from West Africa. Bulletin de l'I.F.A.N., 20 (2) : 460-538.

- 1963a. - The Delphacid species-complex known as Sogata furcifera (Horvath) (Homoptera : Fulgoroidea). Bulletin of entomological Research, 54 (1) : 45-79.

— 1963b. - New Delphacidae (Homoptera : Fulgoroidea) from South America and West Africa. Annals and Magazine of natural History, 13 (6) : 689-700.

— 1964. - Delphacidae from Madagascar and the Mascarene Islands (Homoptera : Fulgoroidea). Transactions of the Royal entomological Society of London, 116 (7) : 131-150.

— 1969. - Delphacidae (Homoptera, Fulgoroidea) from the Sudan Republic. Acta Entomologica Fennica, 26 : 1-79.

— 1972. - A revision of the genus Matutinus Distant (Homoptera, Fulgoroidea, Delphacidae). Bulletin of entomological Research, 61 : 421-461.

— 1978. - Fulgoroidea (Homoptera) from Vietnam. Annals of Zoology, Warsawa, 34, 9 : 207-279.

— 1982. - A tribal classification of the Tropiduchidae (Homoptera : Fulgoroidea), with the description of a new species on tea in Malaysia. Bulletin of entomological Research, $72: 631-643$.

Ghauri M. S. K., 1966. - Revision of the genus Orosius Distant (Homoptera : Cicadelloidea). Bulletin of the British Museum (Natural History), Entomology, 18 (7) : 231-252.

1978. - A new genus and species of Erythroneurini (Hemiptera : Cicadelloidea) causing severe damage to flamboyant trees (Delonix regia) in Mauritius and Reunion. Bulletin of entomological Research, 68 : 203-206.

— 1979. - The identity of Empoasca dolichi Paoli (Hemiptera : Cicadellidae) and description of new species of Empoasca from light-traps near crops in Malawi and Nigeria. Bulletin of entomological Research, $69:$ 343-355.

Heller F. \& Linnavuori R., 1968. - Cicadelliden aus Aethiopien - Stuttgarter Beiträge Zur Naturkunde, 186 : 1-42. 
Ishinara T., 1982. - Some notes on a leafhopper of economic importance Orosius orientalis (Matsumura, 1914) (Hemiptera : Cicadellidae). Applied Entomology \& Zoology, 17 (3) : 364-367.

Kramer J. P., 1962. - New Liberian leafhoppers of the genus Recilia (Homoptera : Cicadellidae : Deltocephalinae). Proceedings of the biological Society of Washington, 75 : 259-268.

Linnavuori R., 1969. - Contribution à la faune du Congo (Brazzaville). Mission A. Villiers et A. Descarpentries 93. Hémiptères Hylicidae et Cicadellidae. Bulletin I.F.A.N., 31, Sér. A (4) : 1129-1185.

- 1975. - Revision of the Cicadellidae (Homoptera) of the Ethiopian Region III. Deltocephalinae, Hecalini. Acta Zoologica Fennica, 143 : 1-37.

— 1977. - Revision of the Ethiopian Cicadellidae (Hemiptera : Homoptera) : Penthimiinae. Etudes du Continent Africain, $4: 5-76$.

— 1979. - Revision of African Cicadellidae (Homoptera : Auchenorhyncha) Part I. Revue de Zoologie africaine, 93 (3) : 647-747.

- 1979. - Revision of the African Cicadellidae (Homoptera : Auchenorhyncha) Part II. Revue de Zoologie africaine, 93 (4) : 929-1010.

Linnavuori R. \& Quartau J. A., 1975. - Revision of the Ethiopian Cicadellidae (Hemiptera : Homoptera) : Iassinae and Acroponinae. Etudes du Continent Africain, 3 : 1-170.

Melichar L., 1898. - Monographie des Ricaniidae (Homoptera). Annalen des Naturhistorischen Museums in Wien, 13, 197-359.

- 1923. - Homoptera, Fam. Acanaloniidae, Flatidae et Ricaniidae. Genera Insectorum, $182,185 \mathrm{pp}$.

Metcalf Z. P., 1957. - General Catalogue of the Hemiptera. Fasc. IV, Fulgoroidea. Part 13, Flatidae and Hypochtonellidae. North Carolina State College, Raleigh, N.C., U.S.A., $565 \mathrm{pp}$.

Oman P. W., Knight W. J. \& Nielson W. W., 1990. - Leafhoppers (Cicadellidae) : a bibliography, generic check-list and index to the world literature 1956-1985. CAB-International Institute of Entomology, $368 \mathrm{pp}$.

Plenet A., 1965. - Parasites animaux des principales plantes cultivées à la Réunion. In: «Congrès de la protection des cultures tropicales. C.R. des travaux. Chambre de Commerce et d'Industrie, Marseille. 23-27/ III/65" : 203-216.

Rensburg van G. D. J., 1983. - South African species of the genus Cicadulina China (Homoptera : Cicadellidae) with descriptions of new species. Entomology Memoir, Department of Agriculture, Republic of South Africa, $57:$ 1-22.

Reynaud B., 1988. - Transmission des virus de la striure, du stripe et de la mosaïque du maïs par leurs vecteurs Cicadulina mbila (Naude, 1924) et Peregrinus maidis (Ashmead, 1980) (Homoptera). Approches biologique, génétique et épidémiologique de la relation vecteur-virus-plante. Thèse Université des Sciences Techniques du Languedoc, Montpellier, $173 \mathrm{pp}$.

Ross H. H., 1968. - The evolution and dispersal of the grassland leafhopper genus Exitianus, with keys to the old world species (Cicadellidae: Hemiptera). Bulletin of the British Museum (Natural History), Entomology., 22 (1) : 1-30.

Roura A., 1977. — La cicadelle du flamboyant. S.P.V.-Réunion-Mayotte. Bulletin d'Information Phytosanitaire, 1, p. 11.

Signoret V., 1860. - Hémiptères de Madagascar. Annales de la Société entomologique de France, 3 (8) : 186.

- 1862. - Maillard, note sur l'île de la Réunion, 2, ann. Journal : 30.

STÅL C., 1866. - Hemiptera Africana, 4. Stockholm, 276 pp. 
Synave H., 1958. - Une famille nouvelle pour la faune des îles de la Réunion et Maurice: les Kinnaridae (Homoptera, Fulgoroidea). Bulletin et Annales de la Société royale entomologique de Belgique, 94 : 118-121.

— 1959. - Cixiidae de la Réunion. Le Naturaliste malgache, 11 : 133-145.

1960. - Contribution à la connaissance des Fulgoroides de l'île Maurice. Bulletin de l'Institut royal des Sciences naturelles de Belgique, 36 (36) : 1-12.

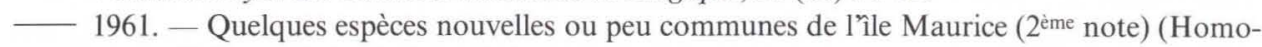
ptera, Fulgoroidea). Bulletin de l'Institut royal des Sciences naturelles de Belgique, 37 (21) : $1-20$.

— 1965. - Contribution à la connaissance des Cixiidae de Madagascar, Maurice et la Réunion (Homoptera-Fulgoroidea). Bulletin de l'Institut royal des Sciences naturelles de Belgique, 41 (27) : 1-57.

Theron J. G., 1975. - Afrosteles, a new genus of South African Macrostelini (Hemiptera: Cicadellidae). Journal of the entomological Society of South Africa, 38 (2) : 207-209.

— 1982. - Grassland leafhoppers (Hemiptera : Cicadellidae) from Natal, South Africa, with descriptions of new genera and species. Phytophylactica, $14: 17-30$.

Webi M. D., 1987. - Species recognition in Cicadulina leafhoppers (Hemiptera : Cicadellidae), vectors of pathogens of Gramineae. Bulletin of entomological Research, 77 : 683-712.

Williams J.R., 1957. - The sugar-cane Delphacidae and their natural enemies in Mauritius. Transactions of the royal entomological Society of London, $109: 55-110$.

— 1975a. - The Cixiidae (Fulgoroidea : Homoptera) of Mauritius. Journal of Natural History, 9 : 169-204.

— 1975b. - Cixiidae (Fulgoroidea : Homoptera) from Reunion Island. Journal of Natural History, 9 : 669-680.

- 1976. - Derbidae (Fulgoroidea) from the Mascarenes. The Mauritius Institute Bulletin, 8 (2) : 129-144.

— 1981. - Tropiduchidae (Fulgoroidea : Homoptera) from the Mascarenes. Journal of the entomological Society of South Africa, 44 (1) : 109-130.

- 1982. - Issidae (Hemiptera : Fulgoroidea) from the Mascarenes. Journal of the entomological Society of South Africa, 45 (1) : 43-56.

Williams J. R. \& Mamet J. R., 1954. - Insects and other invertebrates which feed on sugar cane in Mauritius and Reunion, and their natural enemies. Bulletin of Department of Agriculture Mauritius, Port-Louis, 89 : 1-19.

Williams J. R. \& Fennah R. G., 1980. - Ricaniidae (Hemiptera: Fulgoroidea) from Mauritius, with a description of Trisanor cicatricosus spec. nov., gen. nov. Journal of the entomological Society of South Africa, 43 (1) : 7-22.

Williams J. R. \& Williams D. J., 1988. - Homoptera of the Mascarene Islands. - An annotated catalogue. Entomology Memoir, Department of Agriculture and Water Supply, R.S.A., 72, 98 pp.

Young D. A., 1986. - Taxonomic study of the Cicadellinae (Homoptera : Cicadellidae) Part 3. Old world Cicadellini. Technical Bulletin of the North Carolina State University, Agricultural Research Service, Raleigh, 281 : 1-639. 